\title{
Transfer of Graduating Residents' Continuity Practices
}

\author{
Carol K. Bates, $M D^{7}$, Laurie C. Caines, $M D^{2}$, Diane Brockmeyer, $M D^{7}$, Hans Kim, MD', \\ Anjala Tess, $\mathrm{MD}^{\prime}$, and Gila Kriegel, $\mathrm{MD}^{\top}$ \\ ${ }^{1}$ Beth Israel Deaconess Medical Center, Boston, MA, USA; ${ }^{2}$ University of Connecticut, Famington, CT, USA.
}

$\mathrm{J}$ Gen Intern Med 27(2):146

DOI: $10.1007 / \mathrm{s} 11606-011-1913-4$

(C) Society of General Internal Medicine 2011

Authors Reply: We agree with Drs. Pincavage, Ratner, and Arora that resident patients are indeed often at higher risk for missing appointments. We did not in our study look at the issue of prior behavior with respect to missed appointments, but in a sample of ten patients in our study population, six of the ten had missed several appointments in the years preceding their resident PCP's graduation. In our practice in general, resident patients are more likely to miss scheduled appointments than faculty patients; indeed, resident patients missed $17 \%$ of appointments as compared to $7 \%$ missed appointments for faculty patients in the past year. We suspect that residents were particularly concerned about patients who missed appointments and may have explicitly listed these patients as high risk for that reason. We agree that handoffs in these patients are likely to be more risky than in patients who generally keep appointments and that special outreach efforts should be made to these patients. This is particularly problematic since the graduated residents most familiar with these at-risk patients and their medical problems are no longer available to detect missed opportunities for care such as overdue radiology studies or blood tests.

We analyzed visits to our own emergency room for this sample of ten patients and found that only a single patient had an ER visit within the year after their resident's graduation. We are unable to capture ER visits and admissions to other hospitals and agree that ER utilization would indeed be a useful variable to examine in future studies of this phenomenon.

We agree that a year is a longer than desired interval for followup of many patients and that many of these patients should have had multiple visits during this study year. We set the year as an intentionally long period. We agree that a future study of diabetics in which there are clear guidelines for intervals of visits might indeed look at shorter intervals between visits.

Finally, we were similarly intrigued with the study by Young and colleagues in which case loads were rebalanced, though note that the number of trainees in that program was much smaller. Young described 16 graduating residents having a mean of only 18-22 patients in each psychiatry resident's case load compared to our population of 46 residents. These investigators did not report on patient retention, so it is not clear that rebalancing loads will indeed improve patient follow-up. We have attempted rebalancing of case loads to a small degree for certain target diagnoses for educational reasons, but many of our patients who do follow up return to see a different resident PCP than our assignment due to a variety of factors. We are skeptical as to whether rebalancing will improve retention unless it is coupled with early intensive outreach from a new provider.

Corresponding Author: Carol K. Bates, MD; Beth Israel Deaconess Medical Center, 330 Brookline Ave, Boston, MA 02215, USA (e-mail: cbates@bidmc.harvard.edu). 